\title{
RELACIONES INTERGUBERNAMENTALES Y POLÍTICA SANITARIA EN ARGENTINA EN EL CONTEXTO DE LA CRISIS 2001/3
}

\author{
INTERGOVERNMENTAL RELATIONS AND SANITATION POLICY IN ARGENTINA DURING THE \\ 2001-3 CRISIS
}

Magdalena Chiara ${ }^{1}$

Resumo El trabajo analiza los efectos de la crisis argentina 2001/3, concebida como resultado de un conjunto de 'insuficiencias acumuladas' que se engarzan entre la economía y la política, en la política sanitaria. A diferencia de lo que sucedía en otros sectores, su expresión en el campo de la salud aparece más vinculada a las demandas de los responsables por la gestión de los servicios que a la acción colectiva. La particularidad de tratarse de una agenda 'mediatizada' por distintos actores responsables del gobierno y la gestión del sector exige poner el foco en la trama de las relaciones intergubernamentales resultante de la organización institucional argentina. Este trabajo busca comprender cómo el desafío de la recomposición de la legitimidad estatal frente a la crisis afectó el posicionamiento de los actores gubernamentales en la matriz institucional a partir de la cual se procesa la cuestión sanitaria en Argentina, desde la reconstrucción de las dinámicas y lógicas que convivieron en uno de sus espacios de coordinación intergubernamental: El Consejo Federal de Salud (Cofesa). Desde esta perspectiva y focalizando el análisis en un ejemplo paradigmático como fue el financiamiento de los medicamentos, el Cofesa fue caja de resonancia de estos procesos, conviviendo allí distintas lógicas, algunas generales y otras particulares, del sector salud.

Palavras-chave Política sanitaria; relaciones intergubernamentales; federalismo.
Abstract The present work analyzes the effects of the 2001-3 crisis in Argentina - considered as a result of a set "accumulated insufficiencies" that got entangled in between economy and politics - in the country's sanitation policy. Differently from what was occurring in other sectors, the expression of the crisis in the health sector was more linked to the demands of people responsible for health services management than to collective actions. Both the peculiarity of being an agenda "mediatized" by distinct governmental actors and the health sector management demand focusing on the intergovernmental relations plot resulting from Argentina's institutional organization. The present work aims at understanding how the challenge of rebuilding State legitimacy during the crisis affected the governmental actors position within the institutional framework where Argentina's sanitation issues are dealt with, beginning with the reconstruction of dynamic and logical processes that coexisted in one intergovernmental coordination area: the Federal Health Council (Cofesa, in Spanish). From such perspective and focusing the analysis on a paradigmatic example such as financing of drugs, Cofesa became a touchstone of such processes, where distinct logics of the health sector, some general and some particular, coexist. Keywords sanitation policies; intergovernmental relations; federalism. 


\section{Relaciones intergubernamentales y política sanitaria en el contexto de la crisis 2001/3 en Argentina}

Toda respuesta estatal contiene - casi por definición - elementos vinculados con su eficacia y eficiencia, a la vez que expresa objetivos relativos a la acumulación política y la legitimidad. En las políticas sanitarias en Argentina, esta combinación presenta una complejidad históricamente constituida.

Sobre una trama de competencias constitucionales (ambiguas, difusas y planteadas hasta por defecto) radicadas en la arena provincial pero asumidas 'de hecho' desde el estado nacional hacia mediados del siglo pasado, los recientes procesos de descentralización contribuyeron con el modelado de una matriz compleja y fragmentada de responsabilidades en el diseño y la implementación de la política sanitaria.

La pregunta que orienta este trabajo busca comprender cómo el desafío de la recomposición de la legitimidad estatal frente a la crisis de diciembre de 2001 afectó el posicionamiento de los actores gubernamentales en la matriz institucional desde la que se procesa la cuestión sanitaria en Argentina, reconstruyendo las dinámicas y las lógicas que convivieron en uno de sus espacios de coordinación intergubernamental (el Cofesa).

En un primer apartado se presentan las coordenadas de la trama resultante de la descentralización en el sector salud; en el segundo se presentan los principales atributos que tuvo la crisis cuyo punto más crítico tuvo lugar en diciembre de 2001; un tercer apartado analiza cómo la crisis de legitimidad y los esfuerzos por su recomposición desafiaron a las relaciones intergubernamentales y en el apartado siguiente, se realiza una aproximación al análisis de la dinámica en los espacios de articulación intergubernamental relacionada con el del Consejo Federal de Salud (Cofesa), a partir del caso del financiamiento de los medicamentos. El artículo finaliza recapitulando las contribuciones con relación a la pregunta que da origen a estas páginas.

\section{El escenario previo a la crisis: La trama post descentralización en salud}

La distribución de las competencias relativas a la 'cuestión social' entre niveles gubernamentales en Argentina y la dinámica que asume su ejercicio efectivo, reconoce raíces que se remontan a la conformación del estado nación en la segunda mitad del siglo XIX. Al igual que en otros casos nacionales, en Argentina la Constitución define potestades federales y concurrentes, dejando las competencias no especificadas a cargo de los gobiernos provinciales. ${ }^{2}$ Mientras el gobierno federal tiene responsabilidad exclusiva en política exterior, regulación de la economía y de la defensa, hay potestades compartidas en la justicia, la educación primaria y la seguridad social; en este esquema, toda competencia 
no especificada permanece en la órbita provincial. Se trata de un encuadre constitucional bastante impreciso en lo referente a las responsabilidades que como veremos más adelante - fueron modificadas en las últimas décadas (Cetrángolo y Jiménez, 2004, p. 118-119).

A diferencia de lo que sucede en otros campos, en la política social el ejercicio efectivo de las competencias relativas a los servicios sociales universales (educación y salud) ha sido - en las últimas décadas - objeto de tratamientos diversos. La 'salud' presenta una situación particular ya que, a diferencia de la 'educación', no figuraba en el siglo XIX entre las demandas políticas y no figura tampoco en la Constitución Nacional como objeto de definición de responsabilidad entre los distintos niveles jurisdiccionales. Es recién hacia mediados del siglo XX y como parte de la constitución del Estado Social, que el gobierno nacional avanza 'inconstitucionalmente' 3 con la apertura de hospitales nacionales que fueron posteriormente descentralizados a las provincias. Es notable que tampoco la Constitución sancionada en 1994 haya incorporado a la salud como cuestión de agenda (Centrángolo en Pnud, 2004, p. 59). Dada esta génesis, la 'salud' es una de las cuestiones que - por no haber sido delegadas por los Estados federados al nivel central - permanece en la órbita provincial y ha sido sancionada - de distinto modo - por las constituciones provinciales tanto en sus orígenes como en las reformas recientes (De Marco, 2009).

Sobre esta primaria y también ambigua distribución de competencias, tuvieron lugar las corrientes descentralizadoras, orientadas - como en casi todos los casos - por los principios de eficiencia y por la necesidad de resolver las presiones fiscales de nivel nacional.

La descentralización de los hospitales nacionales se inicia en 1957 y avanza en el año 1978, cuando el gobierno de facto transfiere 65 hospitales nacionales a las provincias (Ley 21.883); tal como se anticipó, estas transferencias estuvieron animadas por imperativos fiscales y no fueron realizadas con la consecuente transferencia de recursos. Aunque esta decisión ayudó a los gobiernos nacionales a alcanzar el objetivo de reducir sus presupuestos, dejó a las provincias en condiciones muy precarias para el sostenimiento efectivo de los servicios.

En un contexto de importantes disparidades regionales, esta transferencia de servicios ha tenido distintos costos en materia de 'cohesión social' encontrándose en el campo de la salud una multiplicación de las respuestas locales, con consecuencias diversas en la calidad, la eficiencia y la equidad en el acceso a los servicios. Tal como sostienen Cetrángolo y Jiménez, "la falta de coordinación desde el nivel central ha permitido una gran heterogeneidad de conductas a nivel provincial y municipal, que responde a grandes rasgos a la configuración previa de cada sistema y a la política sectorial adoptada en cada lugar en respuesta al proceso descentralizador" (Cetrángolo y Jiménez, 2004, p. 119).

Si bien la generación de 'respuestas locales' podría ser considerada como una 'fuerza endógena', que impone desde lo subnacional su lógica al conjunto 
de políticas del bienestar, puede convertirse en un fenómeno virtuoso siempre que encuentre posibilidades de coordinación. La complejidad de la trama de actores responsables de la política sanitaria confiere particular relevancia a estos espacios, particularmente al Cofesa ${ }^{4}$ como ámbito de articulación de las relaciones intergubernamentales, tanto horizontales como verticales.

Como muestra el recorrido realizado hasta aquí, el proceso de descentralización de las últimas décadas complejizó las tensiones en las relaciones entre la nación y las provincias, creando nuevos actores en el nivel subnacional (con niveles desiguales de poder) con dificultades diferenciales para asumir un ejercicio efectivo de los servicios transferidos, fenómeno fuertemente influido por las inequidades existentes entre las provincias.

La dinámica centrífuga de la descentralización parece haber alterado aquel equilibrio en la tensión constitutiva del "sistema federal centralizado" con presidentes fuertes y gobernadores débiles (Faletti, 2004, p. 77-78), rasgo característico del orden conservador en Argentina. Si las elecciones presidenciales tuvieron tradicionalmente un efecto 'arrastre' derivado - en parte - de la capacidad de resolución de demandas desde la estructura estatal nacional, el esquema resultante de la descentralización modificó los mecanismos de equilibrio dotando de distintos recursos de poder a los gobiernos subnacionales.

Este nuevo escenario profundiza un atributo que caracterizó al sector: Las políticas sanitarias son (siguiendo a Eugenio Lahera en su análisis para la relación entre política y política pública en América Latina) “políticas de más de un gobierno, lo que plantea una especificidad política" tanto en su diseño como en su ejecución (Lahera, 2004, p. 10).

Si esta característica complejiza (en cualquier contexto) el diseño y la gestión de la política sanitaria, la crisis sufrida en los primeros años de este milenio y los esfuerzos de los distintos actores responsables por recomponer la legitimidad estatal exacerban su carácter político, aspecto que resulta necesario incorporar en el análisis.

\section{Una aproximación a la crisis argentina de diciembre de 2001}

Apenas celebradas las elecciones del mes de octubre y finalizando el año 2001, Argentina enfrentó una profunda crisis económica, social y política. La economía que venía sufriendo los avatares de los mercados internacionales de capitales desde el año 1998, entra en una fase recesiva que se extiende por tres años, poniéndose en evidencia la crisis del modelo económico basado en la convertibilidad y el colapso de la respuesta estatal frente a la creciente y generalizada acción colectiva (Gómez, 2006, p. 8-12).

En el mes de diciembre de 1999 asumió una fórmula presidencial surgida de la alianza entre la Unión Cívica Radical y el Frente para un País Solidario (Frepaso), 
encabezada por Fernando De la Rúa (UCR) y por Carlos Álvarez (Frepaso), pero los dos años que duró el gobierno tuvieron lugar en un contexto de recesión ininterrumpida y de crisis, fiscal y económica, que se profundiza en el año 2001.

La confiscación de los depósitos bancarios movilizó a las clases medias urbanas a la protesta expresada en los 'cacerolazos' que, combinada con la ola de saqueos a comercios, precipitó la escalada de acción colectiva generalizada que terminó convergiendo en los acontecimientos de diciembre de 2001, la caída del gobierno y la instalación en amplios sectores de la opinión pública de la consigna "que se vayan todos" (Gómez, 2006, p. 12).

Se trató, parafraseando a Botana, de una "condensación de insuficiencias acumuladas":

Lo que ocurrió a fines de 2001 fue en buena medida una súbita condensación de esas insuficiencias acumuladas: insuficiencias en las instituciones, insuficiencias en la economía, insuficiencias en los gobernantes. Parecía que la constitución política del Estado vacilaba ante el descalabro de su economía y que, a la inversa, la propia economía carecía de fiadores y garantes (Botana, 2006, p. 30).

Aunque focalizando el análisis en la capacidad de respuesta del gobierno, Gómez describe:

Las élites políticas y las clases dominantes parecían haberse quedado sin respuestas, vacilando ante dos cuestiones: Cómo parar la movilización de la 'gente en la calle' y su poder destituyente (represión o 'restablecer la confianza en las instituciones') y cómo salir del régimen de convertibilidad (dolarización o devaluación) (Gómez, 1996, p. 12).

La renuncia del Presidente De la Rúa fue sucedida por distintos presidentes designados, culminando el 2 de enero de 2002 con el nombramiento como Presidente de la Nación del Dr. Eduardo Duhalde, senador por la Provincia de Buenos Aires.

El nuevo gobierno enfrentó una situación crítica. El derrumbe del Plan de Convertibilidad, el default en la deuda externa, la inmovilidad de los depósitos bancarios, las altas tasas de inflación, la contracción de la actividad económica y la devaluación del tipo de cambio, impactaron severamente en los sectores medios y en aquellos de menores ingresos.

La caída Plan de Convertibilidad 5 trajo como consecuencia una marcada depreciación del peso y Argentina comenzó a experimentar una significativa inflación por primera vez desde 1991. El brusco descenso de la actividad económica y la depreciación de la moneda agravaron severamente las ya difíciles condiciones sociales del país a partir de una devastadora pérdida de puestos de trabajo. El efecto combinado de todos estos factores produjo una situación social crecientemente conflictiva; la pobreza por ingresos pasó 
del 38,3\% en octubre de 2001 al 53,0\% en mayo de 2002, al tiempo que la indigencia varió del $13,6 \%$ al $24,8 \%$ en el mismo periodo (Fiszbein, Giovagnoli y Adúriz, 2003, p. 150).

En síntesis, el escenario que se abre con la crisis argentina de 2001 está caracterizado por: (i) el fracaso del gobierno de la Alianza por conformar un núcleo de gobernabilidad para sostenerse en el poder; y (ii) la profundización de una crisis económica cuyo alcance se manifestaba en recesión, aumento del desempleo y subempleo, empobrecimiento de la población, pérdida de competitividad y caída del PBI per cápita. El efecto combinado de estos factores produjo cambios abruptos en las condiciones de reproducción de la vida de amplios sectores de la población y, concomitantemente, aceleradísimas transformaciones en los problemas a los cuales dar respuesta.

La pregunta que orienta este análisis se sitúa en el problema de la crisis como 'crisis de legitimidad', en la interfase entre los debates en torno a la legitimidad del poder estatal y aquel campo de la institucionalidad, a través de la cual se da respuesta a las demandas desde la política sanitaria. Este análisis reviste interés dado que las políticas públicas son respuestas a las necesidades de la población y, tal como hemos señalado, también mecanismos de legitimación de la acción estatal; especialmente, en el campo de la política social.

Aunque la coyuntura que estamos analizando presenta rasgos comunes a todos los ámbitos de las políticas, la dinámica que enfrentó el Estado en sus distintos niveles con respecto a la cuestión sanitaria está signada - adicionalmente - por procesos particulares: 6

a) Corte de los servicios de la Seguridad Social, reducción de cobertura de las prepagas e incorporación de 'co-pagos' y desplazamientos de población hacia planes más baratos.

b) Ruptura de la cadena de pagos en el sector (medicamentos, insumos y servicios tercerizados de los hospitales) con sistemas de pasivos prestacionales en los tres niveles de gestión de servicios (nacional, provincial y también municipal). c) Aumento en los costos de medicamentos e insumos biomédicos importados como consecuencia de la devaluación, con implicaciones negativas en materia de abastecimiento y acceso a los mismos (caída de licitaciones públicas, falta de precios como resultado del contexto resultante de la devaluación).

d) Incremento de la demanda de servicios en la red pública, consecuencia de la reducción en la extensión (y efectividad) de la cobertura de planes de salud privados, de la menor capacidad de gasto del bolsillo de los ciudadanos y del deterioro en las condiciones de vida.

e) Mayor riesgo epidemiológico, consecuencia del deterioro en las condiciones socioeconómicas de gran parte de la población (Leptospirosis, Hanta, TBC, Triquinosis, etc.).

f) Demoras en el pago de salarios de los trabajadores del sector salud en las provincias y en los municipios. 
g) Paros, asambleas y reclamos protagonizados por las distintas entidades profesionales del sector en los hospitales que llegan a la presentación de recursos judiciales por parte de entidades gremiales.

h) En particular en el nivel municipal, se advierten cambios en el tipo y urgencia de la demanda. La cuestión alimentaria, los proyectos productivos y la atención a grupos vulnerables desplaza a otras iniciativas que requieren de un mediano plazo como las políticas de salud. La cuestión sanitaria emerge como problema a través de la falta de medicamentos e insumos en los hospitales, protagonizada por los distintos actores que, en las distintas jurisdicciones, tenían a su cargo la prestación de los servicios.

i) En el contexto de este debilitamiento, distintas 'voces' enunciaban la necesidad de supresión de la cartera nacional de salud.

La situación de crisis se institucionaliza con la declaración de 'emergencia sanitaria' en marzo de 2002 con el propósito de dar respuesta inmediata a los problemas que afectaban al sector (Decreto Nacional 486/02).

A diferencia de lo que sucedió en otros sectores de políticas, 7 la cuestión sanitaria no aparecía vinculada a la acción directa de la ciudadanía: Los reclamos y demandas eran enunciados por actores responsables de la gestión de los servicios (ministros, secretarios de salud provinciales y municipales, directores de hospitales, gremios de sanidad). En consecuencia, la inclusión de la cuestión sanitaria en la agenda gubernamental buscaba evitar - en muchos casos - que ésta se 'sumara' a la ya extensa nómina de demandas presentes en la agenda pública.

\section{Legitimidad estatal y crisis en el sector salud: desafíos para las relaciones intergubernamentales (RIGs)}

La cuestión sanitaria no se encontraba en la agenda de las demandas que animaban la acción colectiva en aquellos tiempos. Los registros periodísticos muestran más declaraciones de dirigentes sindicales del ámbito de la salud, planteos de gobernadores y ministros, denuncias judiciales y recursos de amparo presentados por directores de hospitales y por asociaciones gremiales, que reclamos de la ciudadanía frente al colapso de los servicios de salud y a la carencia de insumos y medicamentos. Su presencia en la agenda pública devenía más de la acción pública de estos actores que de demandas planteadas por la ciudadanía.

Las condiciones particulares en que se desenvuelve la cuestión sanitaria, invitan a poner el foco en los impactos de la crisis en la trama de relaciones intergubernamentales entre la nación y las provincias, que resulta de la organización institucional en Argentina.

En aquellos meses, esa "condensación de insuficiencias" (siguiendo a Botana) se advertía muy particularmente en el plano político: “(...) la representación 
temblaba y los argentinos, en un rapto de acalorada frustración, ignoraban en la práctica cómo representarse" (Botana, 2006, p. 21).

Si la crisis anidaba en un particular 'engarce' 8 entre la política y la economía (en su aspecto fiscal y monetario), no puede sorprender que esa particular articulación haya también caracterizado a las estrategias de salida.

La puesta en marcha de la Mesa de Diálogo Argentino fue un intento por construir un espacio desde el cual poner en escena a actores que, aún no siendo parte de la denominada 'nueva política', parecían contar con algo más de aceptación entre la población (Iglesia católica, Pnud, organizaciones sociales y culturales); de ella participó también el Ministerio de Salud de la Nación.

Mientras funcionaba la Mesa de Diálogo Argentino y comenzaba a instalarse (al menos en los medios de comunicación) la agenda de la Reforma Política (Botana, 2006, p. 33), los emergentes de la 'vieja política' seguían siendo protagonistas de una parte importante de las respuestas a las demandas $\mathrm{y}$, a su vez, eran aquellos que sufrían la mayor erosión en la confianza de la ciudadanía. ${ }^{9}$ Coexistían así emergentes de lo 'nuevo' y de lo 'viejo' de la política argentina. 10

La complejidad institucional (y también política) resultante de la descentralización comprometía en los distintos escenarios de articulación intergubernamental a representantes del gobierno nacional, de las provincias $y$, en algunos casos y según el sector de políticas, de los municipios. En esta trama de relaciones convergían - siguiendo a Jordana - relaciones dominadas por tres lógicas diferenciadas. Por una parte, aquellas formalizadas entre niveles de gobierno; en segundo lugar las relaciones resultantes de la coordinación informal entre representantes y responsables de los distintos niveles de gobierno a través de sus partidos políticos; $y$, finalmente, los emergentes de la creación de redes intergubernamentales entre las comunidades profesionales que dirigen y gestionan las políticas sanitarias (Jordana, 2001, p. 29-30). En su dimensión más política, la crisis que estamos analizando y los esfuerzos por recomponer la legitimidad estatal impactaron en las dos primeras lógicas.

Aunque el foco de nuestro análisis está puesto en las políticas de salud, la pregunta en torno de la recomposición de la legitimidad y de los impactos en estas dos lógicas (la institucionalidad formal de las RIGs del sector y la dinámica partidaria que - también - las anima) no puede pasar por alto las características que tuvo la salida institucional a la crisis argentina en esos años.

Sin pretender ahondar en estas páginas en la complejidad con que nos enfrenta este periodo, la propia génesis de la designación de Eduardo Duhaldell como Presidente de la Nación abría un debate explícito acerca de la legitimidad. En un trabajo en el que se analizan las continuidades y discontinuidades a respecto de la lógica previa del sistema político argentino, Novaro hace explícito este problema: “La presidencia de Duhalde estaría signada, desde un comienzo, por un abierto debate a respecto de su legitimidad de origen y el persistente 
reclamo desde distintos sectores, incluso del peronismo, de elecciones anticipadas para todos los cargos, o al menos para presidente" (Novaro, 2004, p. 348).

La dinámica que tiene lugar a partir de este hecho como bien destaca Novaro, se libraba - en simultáneo - en la dinámica intrapartidaria del peronismo y en la posibilidad de acumular por fuera del partido, aunque evitando la fractura.

Los recursos de poder de que dispone el Gobierno nacional en Argentina encuentran 'contrapesos' en los gobiernos provinciales y en la estructura federal de los partidos. De modo tal que - siguiendo también a Novaro -

para garantizar un esquema de gobernabilidad los presidentes se ven obligados a formar coaliciones intrapartidarias con caudillos locales de su propia fuerza y, cuando ello no es suficiente para garantizar mayorías legislativas, coaliciones interpartidarias con otros partidos menores (provinciales o nacionales) que cuenten con representación parlamentaria. Si a ello le sumamos el hecho de que conviven un sistema de partidos muy fluido, es decir poco consolidado, a nivel nacional, con subsistemas provinciales mucho más consolidados y estables, es fácil concluir que para la formación de estas coaliciones los presidentes pueden enfrentar condiciones desfavorables de negociación de cara a gobernadores de otros partidos o incluso del suyo propio (Novaro, 2004, p. 365).

Aunque la arena más evidente en que pueden celebrarse estas coaliciones (al menos en la formalidad de las instituciones de la Constitución) es el Parlamento nacional, no cabe duda que la trama que allí se teje podría impactar en los espacios sectoriales de articulación intergubernamental; abonando así a validar la relevancia que tienen en el análisis de las relaciones intergubernamentales las tres lógicas identificadas por Jordana.

La búsqueda de este punto de equilibrio desafía a los gobiernos en un esquema institucional que combina presidentes fuertes y base electoral y partidaria fragmentada por el federalismo institucional y partidario (Novaro, 2004, p. 366), situación que parece haberse profundizado a propósito de la crisis argentina de diciembre de 2001.12

En este periodo, tuvieron lugar cambios bruscos en la dinámica de 'ida y vuelta' entre los liderazgos del gobierno nacional y de los gobiernos provinciales; tal como describe Botana:

Cuando los liderazgos son débiles (...) y el partido de oposición, dominante a su vez en las provincias, no ejerce el gobierno nacional (...), la acción de los partidos, en ausencia de una conducción centralizada, calza mejor con la imagen difundida en los medios de una 'Liga de Gobernadores'. El sistema de partidos, podríamos decir, se federaliza. Cuando, en cambio, los liderazgos nacionales son fuertes y el tesoro federal que ellos controlan dispone de recursos, los líderes territoriales - poco tiempo antes guardianes celosos de su autonomía - pasan a hacer las veces de gerentes de 
una red de sucursales dependientes de la casa central ubicada en la presidencia. La llamada 'territorialización' de la política tiene que ver con el papel declinante del lazo histórico de subordinación entre presidentes y gobernadores (Botana, 2006, p. 51).

Nos interesa rescatar del aporte de Botana dos aspectos. Por una parte, que este equilibrio inestable es constitutivo de las relaciones entre la nación y las provincias en Argentina y nos permite traer al análisis la idea de legitimidad como 'probabilidad'; y por otra, nos interesa enfatizar el papel que juegan aquellos factores que inciden en la construcción de legitimidad, por fuera de la legalidad formal (liderazgo).

Haciendo referencia más explícita al periodo que analizamos, Botana destaca la existencia de sistemas paralelos, situación - a su juicio - 'agravada' por la descentralización:

Durante los dos años de la década del noventa, hasta llegar a la crisis a principios de este siglo, la Argentina parecía gobernada por dos sistemas paralelos. Por un lado, el régimen presidencial que contempla la Constitución con un Poder Legislativo dividido en dos ramas; por otro, el régimen confederativo de los gobernadores que, cuando les tocaba actuar, lo hacían de hecho en una asamblea no prevista en nuestro ordenamiento constitucional, para fijar el alcance (y sobre todo los condicionamientos) de los llamados pactos fiscales. (...) Esta era, a grandes rasgos, la imagen que presentaba el régimen federal frente a un Estado nacional en default interno y externo (Botana, 2006, p. 199-200).

Desde este análisis, la crisis en el plano económico se 'engarzaba' con la crisis política poniendo en evidencia la existencia de un plano institucional formal y otro (también institucional) regido por la lógica de las relaciones políticas entre los gobernadores y el gobierno nacional.

El despunte de la economía, que tiene lugar en el segundo semestre del año 2002, constituye un factor clave para recuperar aquella primacía del gobierno nacional frente a las provincias y la modalidad de gestión centralizada de los planes sociales jugó un papel central en este 'cambio de timón'. El caso paradigmático lo constituyó el Plan Jefas y Jefes de Hogar Desocupados (PJJHD); se trató de una propuesta nacida en la Mesa de Diálogo Argentino que fue inaugurada en los primeros días del gobierno de E. Duhalde, acompañando la gestión del Gobierno nacional hasta iniciada la presidencia de N. Kirchner. ${ }^{13}$ La escala de su cobertura, la lógica universal de la distribución, el carácter centralizado de su gestión y la naturaleza individual del beneficio contribuyeron con la declinación de la acción colectiva de las organizaciones piqueteras, a la vez que se fue avanzando en una política activa de concesiones (Gómez, 2006, p. 15).

Desde un esquema de gestión centralizado, se vuelven a activar desde el PJJHD las estructuras estatales y las políticas tradicionales en puntos de referencia del acceso a los recursos de parte de las poblaciones pobres; haciendo explícitos 
parte de estos objetivos, la estrategia mediática del programa hacía referencia a "una Argentina de pie y en paz".14

Si reconocemos - tal como ya hemos argumentado - que las relaciones intergubernamentales están animadas por lógicas institucionales formales y también por lógicas que anidan en la dinámica partidaria (tanto intra como interpartidaria), la búsqueda por evitar la declinación de aquel lazo histórico de subordinación entre presidentes y gobernadores parece instalarse en el centro de la trama de las relaciones intergubernamentales. Frente a la necesidad de montar coaliciones con el sistema federal, todas las instancias podrían llegar a ser valiosas (y también válidas).

Sin duda, en la búsqueda de este equilibrio sigue vigente el doble desafío de, por una parte, contener la fractura, y al mismo tiempo abrir el juego a otros sectores fuera del partido peronista (en particular en aquellos partidos que ocupan gobiernos provinciales). A modo de hipótesis, interrogamos a los espacios de articulación intergubernamental que, entretanto, pueden convertirse - en este contexto - en 'cajas de resonancia' o en 'espacios de despliegue' de aquellas dinámicas que, aunque por fuera del análisis del sector salud, están en el centro del funcionamiento del sistema federal argentino.

\section{Las dinámicas en los espacios de articulación intergubernamental en salud15}

Los actores gubernamentales responsables por la política sanitaria en los distintos niveles gubernamentales interactúan en distintos escenarios de coordinación. En el nivel nacional, el escenario más relevante en aquellos tiempos era el Cofesa. Creado por el Decreto 22.373/1980, es un órgano de coordinación sectorial del que participan los responsables por los Ministerios o Secretarías de los Estados federados, presidido por el Ministro de Salud de la Nación. Se trata de un espacio político de concertación de intereses entre los Ministros de Salud y los representantes de los Gobernadores electos de cada provincia.

En los estudios consultados (Rodrigáñez y Tobar, 2003 y Potenza, 2004), se constata que su desempeño estuvo condicionado por la relevancia y el liderazgo de las autoridades sanitarias nacionales, 16 así como por cuestiones relativas a las relaciones políticas entre el gobierno nacional y las provincias. Según estos trabajos ha sido un espacio 'expositivo' o bien 'reclamativo'; la "participación y el diálogo tuvieron lugar por momentos, cuando las condiciones políticas (extra sectoriales) lo permitieron o cuando el debate sanitario lograba hacer aparición" (Rodrigáñez y Tobar, 2003, p. 39).

Aunque se trata de una línea de interpretación posible, a nuestro juicio esta aproximación encuentra ciertas dificultades para comprender la relación entre, por una parte, los alineamientos partidarios que tienen lugar en los espacios de coordinación intergubernamental y, por otra, el debate sanitario. 
Interesa considerar aquí que los acuerdos a los que puedan arribarse en estos espacios de coordinación, no están fundados de manera exclusiva en la racionalidad abstracta (técnico sanitaria en este caso). En ese sentido, y dada la naturaleza de las relaciones entre el gobierno nacional y los gobiernos provinciales que venimos analizando, la conformación de 'bloques' no debería ser concebida como un factor exógeno a la dinámica de la política sanitaria sino, como aquella, una dimensión en la que se libra (también) la construcción de la respuesta estatal frente a la cuestión sanitaria.

Ya iniciado el año 2002,17 el Cofesa cobró particular relevancia pasando a abordar cuestiones relativas al financiamiento del sector o hacia otros temas que atrajeron particularmente la atención, tales como el Plan de Emergencia para la Salud, la política de universalización del acceso a medicamentos y la ejecución en las provincias de los programas nacionales definidos como prioritarios. Los estudios y los actores entrevistados coinciden en destacar la definición de los índices a utilizar para la transferencia de recursos desde la nación hacia las provincias, como uno de los principales logros de ese periodo.

Frente a los gobiernos provinciales, jaqueados en su capacidad de respuesta por la crisis monetaria y fiscal, la agenda se organiza a partir de los programas financiados desde la nación. La disputa pasaba, no solamente por la magnitud de los recursos a ser asignados a cada jurisdicción, sino por mantener la soberanía en la posterior gestión de modo de definir las condiciones de asignación hacia el interior de las provincias.

Los Compromisos Sectoriales (2002 y 2003) son instrumentos que permiten visibilizar, en estos tiempos, los avances en el Cofesa. Más tarde y en un contexto de recuperación, la existencia del Plan Federal de Salud 2003/2007 con el Presidente N. Kirchner será un elemento a destacar en el análisis.

La posibilidad de disponer de recursos financieros por parte del gobierno nacional, que se fue abriendo hacia mediados de 2002, definió un punto de clivaje en la recuperación de aquel equilibrio que caracterizó históricamente la relación entre la nación y las provincias a través de la figura de "presidentes fuertes y gobernadores débiles"' (Faletti, 2004).

Los relatos aluden a una partida asignada por el Ministerio de Economía en los primeros meses de la crisis al sector salud de cuatro millones de pesos que se distribuye entre los estados provinciales en el contexto del Cofesa (CIPPEC, 2008, p. 27). La definición de prioridades para la asignación de estos recursos entre las provincias (muy magros, desde la perspectiva actual), parece haberse constituido en un tema de agenda del Cofesa, aunque - según los relatos - no fue fácil forjar acuerdos entre y con los ministerios provinciales.

Tal como hemos planteado precedentemente, el incremento en los costos de medicamentos como consecuencia de la devaluación tuvo negativas consecuencias en materia de abastecimiento y acceso; la caída de licitaciones públicas y la falta de precios fueron alguno de sus emergentes más inmediatos. En este contexto 
(y por abordar un aspecto paradigmático pero no único), uno de los ejes centrales se concentró en operar sobre el financiamiento de los medicamentos “con el objetivo de reducir el umbral de desembolso que las familias menos pudientes deberían afrontar para poder mantener o restaurar su salud" (CIPPEC, 2008, p. 23).18 Sin ahondar en la génesis y características de las estrategias que se modelaron para responder a este problema, la política de provisión gratuita de medicamentos iba acompañada también de otros instrumentos orientados a intervenir en el mercado, en particular a través de la Ley de Prescripción de Medicamentos por su nombre genérico, Ley 25.469.19 En particular esta estrategia, operacionalizada a través del Programa Remediar, buscaba también 'traccionar' desde un recurso crítico (el medicamento) la organización del sector hacia el fortalecimiento del primer nivel de atención (Centros de Atención Primaria de la Salud - Caps). 20

En este punto vale la pena detenernos, ya que se advierten distintas tensiones en torno a los intereses y agendas de los actores participantes; sucede que aquel acuerdo en torno de la necesidad de priorizar el financiamiento de medicamentos, no alcanzaba a los criterios que el Ministerio nacional asumía para hacer efectiva su distribución.

La priorización de la Atención Primaria de la Salud, eje indiscutido de política pública en contextos de crisis desde una lógica técnica, no parecía expresar ni recoger los problemas que constituían entonces la agenda sanitaria de las provincias (expresada por los Ministros provinciales y los Gobernadores).

En las arenas provinciales (situación constatada en el relevamiento periodístico del periodo), parece prevalecer la 'perspectiva hospitalaria', sostenida también por otros actores del sistema como los Directores de Hospitales y los representantes gremiales. El 'sentido de la urgencia' que supone la demanda hospitalaria le confiere una jerarquía política que termina tensionando con las propuestas que emanaban entonces del Ministerio nacional.

La política se debatía entonces en una tensión definida entre, por una parte, una perspectiva que buscaba abordar un problema estructural aprovechando la 'oportunidad' para fortalecer la Atención Primaria de la Salud (expresada más cabalmente por el Ministerio nacional) y, por la otra, aquella que reducía la política sanitaria a lo hospitalario (por la urgencia que suponía su jerarquía en la agenda pública).

En ese contexto, la capacidad para liderar y gestionar otras relaciones desde fuera del Cofesa parece haber sido decisiva para resolver esta tensión. Nos referimos a cómo jugó (o se hizo jugar) también la presión de los organismos de crédito (en el caso del financiamiento de medicamentos, el Banco Interamericano de Desarrollo (BID) y la Iglesia Católica, a través de Cáritas; el primero como un actor que definía (por fuera del espacio del Cofesa) reglas de juego que fortalecían la lógica técnica y la segunda en un rol de auditoría al que se sumaríaposteriormente - la Cruz Roja en una dinámica que buscaba inscribir la política 
sanitaria en la estrategia de recomposición de legitimidad inaugurada con la Mesa de Diálogo Argentino.

Esta reconstrucción preliminar pone en evidencia que, en este espacio de articulación intergubernamental (el Cofesa) instituido formalmente con la finalidad de articular la política nacional, se fueron procesando los conflictos desde cuatro lógicas diferentes:

- En primer lugar, la lógica técnica de la cual parecen ser portadores los equipos del Ministerio nacional y alguno de sus aliados externos como el BID; las batallas por las reglas de juego a través de las cuales se distribuirían los medicamentos - en este plano - fueron decisivas.

- En segundo lugar, una lógica más directamente vinculada a los problemas de la agenda pública de las provincias y de las demandas originadas en la crisis hospitalaria, sostenida desde los Ministros de salud provinciales. Si bien esta lógica parece estar poco fundamentada desde lo técnico (en particular desde análisis costo-efectivos) se sostenía desde el sentido de la urgencia que primaba en los días de la crisis.

- Una tercera lógica tenía que ver con la necesidad de recuperar la primacía del Estado nacional frente a las provincias, característica del régimen político argentino. Esta lógica estaba 'en cabeza' del Ministro de Salud de la nación y de los Gobernadores. En este sentido, conciliar la primera lógica con la segunda resultó clave en relación a cómo llevar adelante este imperativo.

- Por último, una cuarta lógica se refiere a la necesidad de actualizar esta trama tradicional de las instituciones protagonistas del diseño y gestión de la política sanitaria (erosionada en su consenso social desde la consigna "que se vayan todos"); en esta línea se introduce la movilización de 3000 voluntarios de Cáritas y de la Cruz Roja recorriendo los Centros de Atención Primaria de la Salud como parte del Consejo Asesor Intersectorial, del que también formaban parte otros 'nuevos' actores como Unicef, la Confederación Médica Argentina y la Confederación Farmacéutica Argentina.

En este contexto complejo, constituido tanto desde la coyuntura de la crisis como de la propia historia del sistema federal argentino, la reconstrucción de la legitimidad estatal se libró en la capacidad por 'navegar' y conciliar en propuestas viables estas cuatro lógicas. En la gestión de esta complejidad, algunos factores parecen haber resultado clave:

a) En primer lugar, la recuperación de recursos de parte del gobierno nacional a partir de mediados del año 2002 y la posibilidad de reorientar crédito externo, factor indispensable - como hemos visto - para alinear las relaciones entre la nación y las provincias.

b) El liderazgo del Ministro de Salud de la nación entre los Gobernadores y la existencia de un equipo de cambio. 21

c) Contar con lineamientos estratégicos para orientar las apuestas frente a las oportunidades que la crisis abría y gestionar los conflictos que de ellas se derivaban. 
d) La posibilidad de movilizar - en los espacios de articulación intergubernamental como fue el Cofesa otras relaciones que resultan de una trama de actores más amplia (Iglesia, Cáritas y BID, en el ejemplo que analizamos).

\section{Recapitulando...}

El trabajo buscó comprender cómo el desafío de la recomposición de la legitimidad estatal frente a la crisis de diciembre de 2001 afectó el posicionamiento de los actores gubernamentales en la matriz institucional desde la que se procesa la cuestión sanitaria en Argentina, a partir de la reconstrucción de las dinámicas y lógicas que convivieron en uno de sus espacios de coordinación intergubernamental (el Cofesa); para ello, pasó revista a la situación que rodeó la puesta en marcha de un programa de provisión gratuita de medicamentos en los Caps.

A la complejidad propia de una situación de crisis, la distribución de competencias en salud en Argentina incorpora una variable previa: la de tratarse de una competencia no delegada por las provincias a la nación, pero de haber sido objeto de una fuerte intervención nacional hacia mediados del siglo XX, tempranamente descentralizada en las provincias. Este origen le confiere ambigüedad relativa al mapa de distribución de las competencias, sobre el que se delinean las relaciones políticas entre los niveles de gobierno.

Estos atributos exigen conceptualizar las relaciones informales como vías de construcción de legitimidad; desde esta perspectiva, la legitimidad como 'probabilidad' se convierte en una tarea permanente de la acción estatal en el diseño y la implementación de la política sanitaria. En una trama institucional como la argentina, la crisis de legitimidad y su reconstrucción afecta no sólo al Presidente sino también a un conjunto bastante más vasto de actores, en particular a aquellos con responsabilidad de gobierno en el nivel subnacional, situación que se profundiza con la descentralización.

El periodo sobre el cual centramos nuestro análisis, la crisis que alcanza su punto más crítico en diciembre de 2001, es expresión de un conjunto de 'insuficiencias acumuladas' que 'se engarzan entre la economía y la política'. Su expresión en la salud, aparece más vinculada a demandas de los responsables de la gestión de los servicios (desde ministros hasta representantes gremiales) que a la acción colectiva.

La particularidad de tratarse de una agenda 'mediatizada' por distintos actores responsables por la gestión del sector en los distintos niveles, llevó a poner el foco en la trama de las relaciones intergubernamentales resultante de nuestra organización institucional. Adicionalmente, cabe destacar que estos actores formaban parte de la entonces denominada 'vieja política', aspecto nada desdeñable si recordamos los 'sentidos'. 
En este campo, distintos procesos que formaron parte de la crisis y de su salida deben reconocerse al abordar la dinámica de las relaciones entre niveles de gobierno en el sector salud. El primero tiene que ver con que la crisis encuentra muy debilitada aquella supremacía del gobierno nacional frente a los gobiernos provinciales, característica del federalismo argentino; el gobierno nacional designado por la legislatura en los primeros días de enero de 2002, atraviesa varios meses hasta que recupera los recursos (financieros pero también institucionales) necesarios para reorientar la relación con los gobernadores. Asimismo, se advierte la importancia de integrar en el análisis de lo sucedido en aquellos meses, a otras dimensiones de la legitimidad que trasciendan aquella basada en la legalidad.

Desde esta perspectiva y focalizando el análisis en un ejemplo paradigmático como fue la provisión gratuita de medicamentos en los establecimientos públicos de salud, el espacio más relevante de articulación de las relaciones intergubernamentales - el Cofesa - fue caja de resonancia de estos procesos y convivieron allí varias lógicas, algunas generales y otras particulares del sector.

Una lógica técnica que buscaba darle viabilidad a un conjunto de reglas de juego para la asignación del recurso 'medicamentos' para responder a la demanda, produciendo - en simultáneo - el fortalecimiento del primer nivel de atención (Caps) y traccionando las consultas desde los hospitales hacia los Caps. En tensión con ésta, una segunda lógica, protagonizada por los Gobernadores provinciales y sus Ministros, expresaba de manera directa la agenda pública asociada a la gestión hospitalaria.

La resolución de esta tensión se complejizaba a partir de la necesidad del gobierno nacional de recuperar la primacía en la relación con las provincias: Responder a sus demandas buscando realizar parte de los objetivos que se habían trazado en la política sanitaria, constituía el desafío mayor y ponía en juego su liderazgo sectorial.

Esta trama en la cual se desplegaban estas relaciones y conflictos era parte de los denominados 'viejos actores'; gobernadores, ministros y secretarios de estado, formaban parte de aquel elenco sancionado desde la consigna 'que se vayan todos'; en ese sentido, la política debía - a su vez - abrirse a los 'nuevos aires' del espíritu de la llamada Mesa de Diálogo Argentino: En esa línea Cáritas y la Cruz Roja entran en escena a partir de propuestas que buscan innovar en formas de auditoría social.

En la complejidad que supone conciliar estas lógicas tan diferentes, se jugaba la recomposición de la legitimidad estatal; en este proceso, el liderazgo, los recursos materiales, contar con una estrategia para el sector y con 'equipos de cambio', parecen haber sido factores cruciales para lograr aquella recomposición que, a juicio de quien escribe y considerando los hechos recientes, tiene aún final abierto. 


\section{Notas}

${ }^{1}$ Investigadora docente. Instituto del Conurbano de la Universidad Nacional de General Sarmiento, Buenos Aires, Argentina. Licenciada en Ciencias Antropológicas y candidata a doctora en Ciencias Sociales por la Universidad de Buenos Aires. <mchiara@ungs.edu.ar> Correspondência: Universidad Nacional de General Sarmiento, Juan M. Gutiérrez 1150, Los Polvorines, Provincia de Buenos Aires, Argentina.

2 Cabe aclarar que las provincias se corresponden con los niveles estaduales de otros países federales de la región.

${ }^{3}$ Retomamos intencionalmente el término utilizado por O. Cetrángolo (Pnud, 2004).

4 Se trata de un espacio de coordinación intergubernamental del cual participan los Ministros de Salud provinciales y el Ministro responsable por la Nación

5 Desde el año 1991 este Plan implicó, además de un severo ajuste fiscal y la decisión de avanzar en las privatizaciones, un nuevo régimen cambiario fijando el tipo de cambio en 10.000 australes igual a 1 dólar estadounidense, y posteriormente, en 1992, en 1 peso argentino igual a 1 dólar estadounidense. Asimismo, se dispuso que el 100\% de los billetes y monedas en circulación debían estar respaldados por reservas en divisas del Banco Central, al cual se obligaba a comprar o vender la cantidad de divisas que el mercado solicitara.

6 Se sigue y profundiza el análisis ya elaborado en Chiara y Jiménez (2007).

7 En este aspecto, se advierten fuertes diferencias con las demandas en torno a la pérdida de puestos de trabajo, protagonizados por las organizaciones de trabajadores desocupados.

8 Tomamos el concepto del texto ya citado de Botana (2006).

9 “(...) el trofeo de la confianza se lo llevan aquellos que hablan, escriben y critican, sin tener responsabilidad de gobierno" (Botana, 2006, p. 61).

10 El uso de las 'comillas' tiene implícito nuestro interés por hacer explícito que se trata de una noción acuñada en otros ámbitos, al mismo tiempo que relativizar su validez.

11 Desde la 'mesa de tres patas' a las que hace referencia Cavarozzi: los gobernadores justicialistas, el Parlamento y el apoyo de Alfonsín y el radicalismo (citado por Botana, 2006, p. 39).

12 Frente a la profundización de este desequilibrio se formulan distintas tesis (algunas más centradas en la salud de las maquinarias partidarias y otras en la lealtad de los electores). No parece pertinente, aunque resulta muy interesante y relevante el debate, avanzar en esta línea de reflexión.

13 Se trata de un programa de subsidios monetarios a cambio de trabajo.

14 Ver Roca (2003) y Andrenacci y otros (2006).

15 Las reflexiones aquí presentadas resultan de las entrevistas realizadas a funcionarios en el marco de la investigación, así como de la lectura (preliminar) de las actas del Cofesa y de las fuentes hemerográficas relevadas. 
16 Cabe destacar que el Ministro Ginés González García estuvo a cargo de la cartera sanitaria nacional en el gobierno de E. Duhalde (2001/2003) y de N. Kirchner (2003/2007).

17 Cabe destacar que las referencias al Cofesa son indirectas ya que recién a partir mediados del año 2003 se dispone de las actas del organismo.

18 Más allá de los estudios existentes sobre el Programa Remediar, interesa recatar de esta publicación las reflexiones del Jefe de Gabinete de Ministros de ese periodo, F. Tobar.

19 La prescripción de medicamentos por su nombre genérico se implementó como modo de hacer frente a la necesidad de acercar la oferta de medicamentos a la demanda de la población en un contexto de fuerte caída de los ingresos reales y el aumento del desempleo. La norma qu el instituye establece que toda prescripción médica debe efectuarse expresando el nombre genérico del medicamento, seguida de forma farmacéutica, cantidad de unidades por envase y concentración. De este modo, el profesional farmacéutico puede informar al paciente acerca de las marcas comerciales que contengan el mismo principio activo indicando en cada caso los distintos precios de estos específicos. De este modo, el paciente puede elegir la marca y precio del medicamento prescripto por el médico, no autorizando la sustitución de la droga prescripta por el médico. (Ministerio de Salud de la Nación, 2009)

20 El Programa Remediar es un programa masivo de provisión gratuita de medicamentos esenciales ambulatorios; fue la estrategia central del Ministerio de Salud de la Nación para enfrentar la emergencia social y sanitaria, garantizando el acceso de la población más vulnerable a los medicamentos esenciales que dan respuesta a la mayoría de los motivos de consulta médica en los Caps. Los objetivos del programa son: “(a) Asegurar a la población sin cobertura de salud y en situación de pobreza el acceso a los medicamentos esenciales; (b) Fortalecer un modelo de atención primaria y promover políticas saludables con gestión participativa." A su vez, el programa asumió los siguientes propósitos: "Jerarquizar los Centros de Atención Primaria de Salud (Caps) fortaleciendo las redes de APS. Complementar la Política Nacional de Medicamentos en la promoción del acceso y utilización de los medicamentos por su nombre genérico. Promover el uso racional de los medicamentos. El programa estaba orientado a la población que está bajo línea de pobreza o no tiene cobertura de obra social" (Ministerio de Salud de la Nación, 2009).

21 Según Belmartino (2008) “el 'equipo de cambio' es el grupo particular de agentes del Estado responsable de formular y promover el cambio de política El supuesto subyacente de estos estudios es que los decisores tienen una agenda política con contenidos propios que no está basada solamente en las presiones de los grupos de interés de la sociedad". 


\section{Referências}

ANDRENACCI, Luciano et al. La Argentina de pie y en paz: acerca del Plan Jefes y Jefas de Hogar Desocupados y del modelo de política social en la Argentina contemporánea. En: ANDRENACCI, Luciano (Comp.). Problemas de política social en la Argentina contemporánea. Buenos Aires: UNGS/Prometeo Libros, 2006, p. 181-212.

BELMARTINO, Susana. La gestión local de atención médica abordada como objeto de investigación. En: CHIARA, Magdalena et al. Gestión Local en Salud: conceptos y experiencias, Buenos Aires: UNGS, 2008, p. 129-141.

BOTANA, Natalio. Poder y hegemonía. El régimen político después de la crisis. Buenos Aires: Emecé, 2006.

CETRÁNGOLO, Oscar y JIMÉNEZ, Juan Pablo. Las relaciones entre niveles de gobierno en Argentina. Revista de la Cepal, Santiago de Chile, v. 84, p. 117-134, diciembre 2004.

CHIARA, Magdalena y JIMÉNEZ, Carlos. Relaciones intergubernamentales (RIGs) y política de salud en el nivel local. El caso del subsector público en el Gran Buenos Aires. En: CONGRESO INTERNACIONAL DEL CLAD SOBRE LA REFORMA DEL ESTADO Y DE LA ADMINISTRACIÓN PÚBLICA, XII, 2007. Santo Domingo: CLAD. 1 CD ROM.

CIPPEC. Experiencias de gestión pública en salud. Primer ciclo. Buenos Aires: Cippec, 2008.

CLEMENTE, Adriana y GIROLAMI, Mónica (Ed.). Memoria de la crisis (II). Buenos Aires: Espacio/IIED-AL, 2006,

DE MARCO, Magdalena. Competencias de Salud Provinciales. Buenos Aires: mimeo., 2009.

GOMEZ, Marcelo. Crisis y recomposición de la respuesta estatal a la acción colectiva desafiante en la Argentina 1989-2004. Revista Argentina de Sociología, Buenos Aires, v. 4, n. 6, p. 88-128, 2006.
FALETTI, Tulia. Federalism and Decentralization in Argentina: Historical Background and New Intergovernmental Relations. En: TULCHIN, Joseph; SELEE, Andrew (Eds.). Decentralization and Democratic Governance in Latin America. Washington, D.C.: Woodrow Wilson Center for Scholars, 2004, p. 67-100.

FISZBEIN, Ariel; GIOVAGNOLI, Paula y ADÚRIZ, Isidro. El impacto de la crisis argentina en el bienestar de los hogares, Revista de la Cepal, Santiago de Chile, n. 79, abril, 2003

JORDANA, Jacint, Relaciones intergubernamentales y descentralización en América Latina: una perspectiva institucional. Indes/ BID, Serie Documentos de Trabajo I-22UE, Washington, D.C., 2001. Disponible en: $<$ www.indes.iadb.org $>$.

LAHERA, Eugenio. Politica y Politicas Públicas. Serie Políticas Sociales, Cepal, Santiago de Chile, n. 95, 2004.

MINISTERIO DE SALUD DE LA NACIÓN. Política Nacional de Medicamentos. Programa Remediar. Disponible en: <www.msal.gov. $\mathrm{ar} / \mathrm{htm} /$ site/Genericos/site2/medidas.asp\#2>. Acceso en: julio 2009.

NOVARO, Marcos. Continuidades y discontinuidades tras el derrumbe político. Revista Saap, Buenos Aires, v. 1, n. 2, p. 341-376. 2004.

POTENZA, Fernanda. Coordinación intergubernamental en politicas sociales: el funcionamiento de los Consejos Federales de Educación y Salud en la elaboración de políticas sectoriales (1990-2004). Disertación Tesis de Maestría en Administración y Políticas Públicas. Buenos Aires: Universidad de San Andrés. 2004.

PROGRAMA NACIONES UNIDAS para el DESARROLLO. La construcción del federalismo argentino: perspectivas comparadas. Buenos Aires. Buenos Aires: Pnud, 2004.

ROCA, Emilia. Plan Jefes y Jefas de Hogar Desocupados. ¿Política de empleo o política 
social?, En: CONGRESO ASET, IV, 2003.

1 CD ROM.

RODRIGÁÑEZ RICCHIERI, Patricia y TOBAR, Federico. El Consejo Federal de Salud (CO.FE.SA). Actor clave en la construcción de un federalismo sanitario efectivo. Buenos Aires: Ministerio de Salud. Presidencia de la Nación. 2003.

Recebido em 28/05/2009

Aprovado em 15/07/2009 